\title{
A Case of Multiple Nodules in a Patient with Lung Cancer - A Diagnostic Challenge
}

\author{
Inês $S \mathrm{P}^{1 *}$, Margarida $\mathrm{S}^{2}$, Margarida $\mathrm{D}^{3}$, Joana $\mathrm{C}^{4}$, Luís $\mathrm{C}^{5}$, José $\mathrm{C}^{5}$, Tiago $\mathrm{P}^{6}$ and Barroso $\mathrm{A}^{7}$ \\ ${ }^{1}$ Resident in Pulmonology, Centro Hospitalar Vila Nova de Gaia/Espinho, Portugal \\ ${ }^{2}$ Resident in Pulmonology, Centro Hospitalar Vila Nova de Gaia/Espinho, Portugal \\ ${ }^{3}$ Assistant in Pulmonology, Thoracic Tumours Multidisciplinary Unit, Centro Hospitalar de Vila Nova de Gaia/Espinho, Portugal \\ ${ }^{4}$ Assistant in Radiation Onchology, Instituto Português de Oncologia do Porto, Portugal \\ ${ }^{5}$ Institute of Molecular Pathology and Immunology of the University of Porto (IPATIMUP), Portugal \\ ${ }^{6}$ Assistant in Radiology, Functional Unit of Intervention Radiology, Centro Hospitalar Vila Nova de Gaia/Espinho, Portugal \\ ${ }^{7}$ Graduated Assistant in Pulmonology, Thoracic Tumours Multidisciplinary Unit, Centro Hospitalar de Vila Nova de Gaia/Espinho, Portugal
}

*Corresponding author: Inês S P, Resident in Pulmonology, Centro Hospitalar Vila Nova de Gaia/Espinho, Portugal.

To Cite This Article: Inês S P, Margarida S, Margarida D, Joana C, Luís C, et al., A Case of Multiple Nodules in a Patient with Lung Cancer - A Diagnostic Challenge. Am J Biomed Sci \& Res. 2021 - 14(2). AJBSR.MS.ID.001966. DOI: 10.34297/AJBSR.2021.14.001966.

Received: 誹 September 07,2021; Published: 割 September 16, 2021

\begin{abstract}
In a patient with diagnosis of lung cancer, the appearance of multiple nodules during follow-up raises various differential diagnosis. The treatment strategy may be different depending on its diagnosis, so the correct approach is crucial. We present a case report of a 69-year-old male that was diagnosed with a lung adenocarcinoma stage IB in 2008 that underwent right upper lobectomy and adjuvant chemotherapy. Ten years later, during follow-up, 6 new nodules appeared on CT scan, 5 of them were biopsied. Four of these nodules were adenocarcinoma and one had histological features of atypia. Only 2 nodules showed similar molecular characteristics, being all the other nodules different from each other. It was assumed they were three synchronous pulmonary neoplasms (the 2 nodules similar to each other were assumed to have the same origin) and patient underwent stereotactic body radiation therapy (SBRT) of all malignant lesions followed by additional chemotherapy. Three years later, there were no signs of relapsing. This clinical case shows the challenge that patients with multiple nodules can be and highlights the importance of a multidisciplinary discussion in the decision of diagnostic and therapeutic approach.
\end{abstract}

Keywords: Lung cancer; nodules; multiple primary lung cancer; staging; molecular characteristics

Abbreviations: COPD-chronic obstructive pulmonary disease; CT - computed tomography; MPLC - multiple primary lung cancer; MRI - magnetic resonance imaging; PD-L1 - programmed death-ligand 1; 18-FDG PET-18-fluorodeoxyglucose positron emission tomography; NGS - next generation sequencing; PS- performance status; SBRT - stereotactic body radiation therapy.

\section{Introduction}

Patients with lung cancer should always maintain clinical and imaging follow-up because there is a possibility of relapse or appearance of different tumours, even when they undergone potentially curative treatment [1]. During follow-up, other pulmonary nodules can be detected, and if so, several questions arise: Is it malignant? Is it a relapse of the first tumour? Is it a new primary tumour? Is it metastases of another primary? To make this differentiation, histological and molecular characterization is essential.

\section{Case Report}

Sixty-nine-year-old male patient, former smoker for 18 years, with a personal history of diabetes mellitus, dyslipidaemia and COPD. In 2008, he was diagnosed with lung adenocarcinoma stage 
IB - cT2aN0M0(lesion 1) and underwent right upper lobectomy plus adjuvant chemotherapy. In 2018, patient remained asymptomatic and with PS 0. During follow-up exams, 5 new nodules (lesions 2-6) were identified in thoracic CT. 18-FDG PET was performed, showing only high 18-FDG uptake in lesion 3 (maximum SUV 4.8). Brain MRI showed no alterations suggestive of metastases. The case was discussed in a multidisciplinary meeting and it was decided to perform histological characterization of all pulmonary lesions by transthoracic biopsy. Lesion 2 presented atypia in two different biopsies while the remaining lesions were adenocarcinoma. NGS and PD-L1 expression was tested in lesion 1 (archived sample) and in the samples of lesions 3-6. NGS showed that only lesion 4 and 6 shared the same molecular profile. To note that these two lesions had ground glass features on thoracic CT and lepidic features on histological characterization. All other lesions were distinct from each other, as we can see in (table 1). Lesion 3, 4 and 6 had KRAS mutation but the site of this mutation was different in lesion 3 , as we can see in (Figure 1).

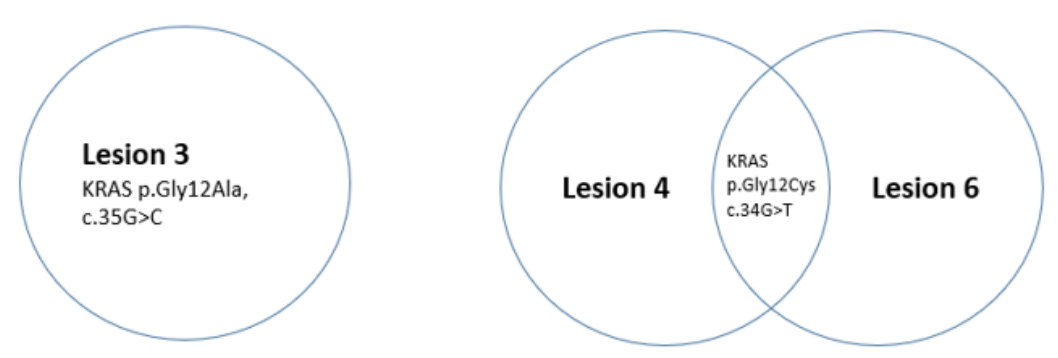

Figure 1: KRAS mutation in lesions 3,4 and 6 .

Table 1: Different characteristics of the nodules.

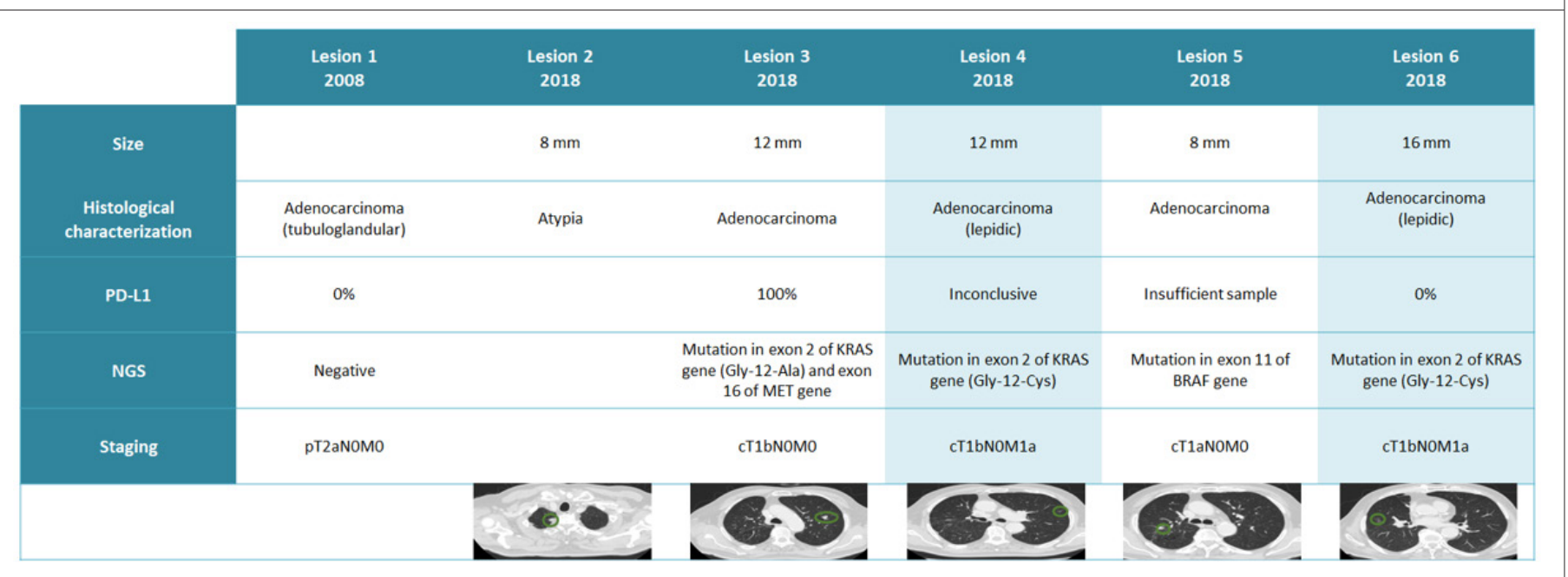

In the meantime, a new $6 \mathrm{~mm}$ nodule was identified in thoracic CT (lesion 7), but it was decided not to biopsy due to its small dimension. In multidisciplinary meeting, it was assumed that these cancers were MPLC, lesion 3 was staged as cT1bN0M0, lesion 4 and 6 as cT1bN0M1a and lesion 5 as cT1aN0M0. It was decided to perform SBRT in lesions 3,4,5 and 6 followed by chemotherapy (carboplatin plus pemetrexed) as lesion 4 and 6 were staged as IVA. Lesions 2 and 7 were kept under surveillance. Three years after the first detection of the new nodules, patient remained under surveillance and there were no signs of relapsing or growth of lesions 2 and 7. After SBRT, patient presented grade 1 radiation pneumonitis that also remained unchanging.

\section{Discussion}

The identification of synchronous MPLC is relatively rare but has increased in recent years [2] probably secondary to the increase use of thoracic CTs. Our patient presented 6 new nodules after 10 or more years of follow up after his surgical treatment to a lung adenocarcinoma, of which 4 were proven to be malignant. This highlights the importance of maintaining the follow up of these patients for a long time, especially if they have risk factors such as active or past smoking. The best surveillance strategy in patients not actively receiving cancer-directed therapy is still on debate [3]. The second aspect we would like to emphasize is the 
diagnostic challenge that the existence of new multiple nodules in patients with a history of a previous lung cancer could be, as they may correspond to MPLC, metastases or benign lesions which has prognostic and therapeutic implications. There is no doubt that the first step should be histological characterization. If we identify a different histology, we can consider a second primary tumour [4].

However, in our patient, lesions 3-6 had histological characteristics of lung adenocarcinoma, like the initial treated lung cancer (lesion 1). Still, the same histologic type does not by itself establish that the lesions are the same tumour [4]. The IASLC Staging and Prognostic Factors Committee tried to developed criteria for clinical and pathologic identification of synchronous separate versus related pulmonary tumours [4]. Relative arguments that favour separate tumours are: 1) different radiographic appearance or metabolic uptake; 2) different pattern of biomarkers (driver gene mutations); 3) different rates of growth (if previous imaging is available); 4) absence of nodal or systemic metastases [4]. In our clinical case, the new synchronous nodules presented different characteristics on CT (lesions 4 and 6 with ground glass features and other lesions with solid pattern), different metabolic uptakes (only lesion 3 had high 18-FDG uptake), different pattern of biomarkers and there was no nodal or systemic metastases. All of this characteristics favour the diagnosis of separate tumours. Nevertheless, if we had just considered the fact that they had the same histological type, we could have misdiagnosed a metastatic relapse of the first lung cancer.

In recent years, one of the most important developments in lung cancer was the improvement in its molecular and genetic characterization, enabling a more precise diagnosis. In fact, as we stated before, the pattern of biomarkers may play a key role in the differential diagnosis of multiple nodules detected by CT [5]. In our clinical case, NGS analysis and PD-L1 determination showed different results in different nodules, with the exception of lesions 4 and 6 that had the same driver gene mutation. This was another argument in favour of assuming a diagnosis of MPLC and also enhanced the probable identical origin of lesion 4 and 6. However, it is not completely clear if the differences in specific mutations of the different nodules identifies separate primary cancers or if they are a consequence of tumoral heterogeneity, as different genetic mutations may coexist in the same tumour [6]. Even metastases can present mutational profiles different from the original tumour [6]. More studies are needed to clarify this issue.

Another interesting point of this clinical case is the staging of lesions 4 and 6 . We assumed they were the same tumour as they had the same radiographic appearance and the same driver gene mutation and we classified them as cT1bN0M1a. However according to TNM $8^{\text {th }}$ edition classification, these lesions can be categorized as multifocal lung adenocarcinoma with ground glass/lepidic features. According to The IASLC Lung Cancer Staging Project, tumours should be categorized as multifocal lung adenocarcinoma with ground glass/lepidic features if there is a malignant subsolid nodule and other nodules with ground glass features, regardless of performing a histological characterization in the other lesions, and if a biopsy is performed, regardless of being lepidic-predominant adenocarcinoma, minimally invasive adenocarcinoma, or adenocarcinoma in situ. [7]. Although the authors highlight that there are frequently 3 to 10 nodules with ground glass appearance on CT scan or lepidic characteristics on histology [7], in our case there was only 2 nodules with this features. Assuming this classification, lesion 4 and 6 could be staged as cT1b(2)N0M0 [7] instead of cT1bN0M1a. Nevertheless, this TNM classification only has the objectives of classify anatomic tumour extent, elucidate the prognosis and facilitate the discussion of how to treat patients, but do not define the treatment of the patients. There is a lack of high-level evidence for standard treatment of multifocal lung adenocarcinoma and the decision of local and systemic treatment is highly variable between specialists [8].

Last but not least, it is important to underline that decision regarding how to categorize multiple lesions in a patient should be made within a multidisciplinary board, considering all information [4]. In fact, most of the above mentioned criteria can be suggestive of separated lung tumours, but are associated with potential misclassification. As a result, some questions arise that are not still answered in the existent literature: Is it appropriate to make the diagnosis of MPLC based on the mutational profile? Is it worthwhile to wait for histological characterization, at the risk of delaying treatment? What is the best strategy to differentiate different pulmonary nodules with accuracy? On the other hand, if we do not perform a histological and molecular characterization of all lesions, and assume that they are pulmonary metastases, the therapeutic decision will probably be different, with no curative intent and potential impact on prognosis. For instance, in our clinical case, pembrolizumab would be an option as first line treatment according to ESMO guidelines [9] if we had only biopsied the single positive PET lesion - lesion 3 (lung adenocarcinoma, PDL1 100\%, no targetable mutations), and assume that all the other nodules were metastatic.

\section{Conclusion}

The identification of multiple nodules is a real diagnostic challenge, and its correct diagnosis and staging has therapeutic and prognostic implications. Thus, it is essential to improve scientific evidence for the diagnostic and therapeutic approach of these nodules that will allow the creation of international consensus. For the time being, our decisions should be based on all available 
information of the multiple nodules, including imaging, biopsy, and clinical features, discussed in a multidisciplinary board. Furthermore, sharing the experience of different teams worldwide is essential.

\section{References}

1. Romaszko AM, Anna Doboszyńska (2018) Multiple primary lung cancer: A literature review. Adv Clin Exp Med 27(5): 725-730.

2. Hongtao Tie, Jun Luo, Rui Shi, Zhenhan Li, Dan Chen, et al. (2020) Characteristics and prognosis of synchronous multiple primary lung cancer after surgical treatment: A systematic review and meta-analysis of current evidence. Cancer Med 10(2): 507-520.

3. Schneider BJ, Nofisat I, Joachim A, Caroline C, Megan ED, et al. (2020) Lung Cancer Surveillance After Definitive Curative-Intent Therapy: ASCO Guideline. J Clin Oncol 38(7): 753-766.

4. Frank CD, Wilbur AF, Andrew GN, Nicolas G, Douglas AA, et al (2016) The IASLC Lung Cancer Staging Project: Background Data and Proposed Criteria to Distinguish Separate Primary Lung Cancers from Metastatic Foci in Patients with Two Lung Tumors in the Forthcoming Eighth
Edition of the TNM Classification for Lung Cancer. J Thorac Oncol 11(5): 651-665.

5. Xiaohui Wang, Yuan Gong, Jianfei Yao, Yan Chen, Yuemin Li, et al. (2021) Establishment of Criteria for Molecular Differential Diagnosis of MPLC and IPM. Front Oncol 10: 614430

6. Dagogo JI, Shaw AT (2017) Tumour heterogeneity and resistance to cancer therapies"; Nature Reviews Clinical Oncology 15(2): 81-94.

7. Frank CD, Wilbur AF, Andrew GN, Nicolas G, Douglas AA, et al (2016) The IASLC Lung Cancer Staging Project: Summary of Proposals for Revisions of the Classification of Lung Cancers with Multiple Pulmonary Sites of Involvement in the Forthcoming Eigth Edition of the TNM Classification. J Thorac Oncol 11(5): 639-650.

8. Konstantinos L, Tobias P, David EM, Julian RM, Shanda Blackmon, et al. (2017) Management of Multifocal Lung Cancer: Results of a Survey. Journal of Thoracic Oncology 12(9): 1398-1402.

9. Planchard D, Popat S, Kerr K, Novello S, Smit E F, et al. (2018) Metastatic non-small cell lung cancer: ESMO Clinical Practice Guidelines for diagnosis, treatment and follow-up. Annals of Oncology 29(Suppl 4): 192-237. 